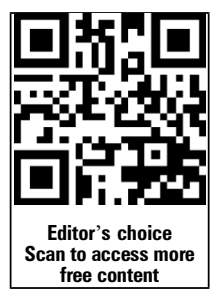

${ }^{1}$ Department of Medical Gerontology, Trinity College Dublin, Dublin, Ireland ${ }^{2}$ Department of Emergency Medicine, St. James's Hospital, Dublin, Ireland

${ }^{3}$ Department of Pharmacology and Therapeutics, Trinity

College Dublin, Dublin, Ireland

${ }^{4}$ Falls and Blackouts Unit,

St. James's Hospital, Dublin, Ireland

${ }^{5}$ Department of Cardiology, St. James's Hospital, Dublin, Ireland

${ }^{6}$ National Heart and Lung Institute, Imperial College London, London, UK

Correspondence to Dr Jaspreet Bhangu, Department of Medical Gerontology, Trinity College Dublin, Dublin 2, Ireland; jaspreetbhangu@gmail.com

Received 15 September 2015 Revised 11 November 2015 Accepted 3 January 2016 Published Online First 28 January 2016

\section{SLinked}

- http://dx.doi.org/10.1136/ heartjnl-2016-309274

\section{CrossMark}

To cite: Bhangu J McMahon CG, Hall P, et al. Heart 2016;102:681-686.

\title{
Long-term cardiac monitoring in older adults with unexplained falls and syncope
}

\author{
Jaspreet Bhangu, ${ }^{1}$ C Geraldine McMahon, ${ }^{2}$ Patricia Hall, ${ }^{1}$ Kathleen Bennett, ${ }^{3}$ \\ Ciara Rice, ${ }^{4}$ Peter Crean, ${ }^{5}$ Richard Sutton, ${ }^{6}$ Rose-Anne Kenny ${ }^{1}$
}

\section{ABSTRACT}

Aims Unexplained falls account for $20 \%$ of falls in older cohorts. The role of the implantable loop recorder (ILR) in the detection of arrhythmias in patients with unexplained falls is unknown. We aimed to examine the diagnostic utility of the ILR in detection of arrhythmogenic causes of unexplained falls in older patients.

Methods A single centre, prospective, observational cohort study of recurrent fallers over the age of 50 years with two or more unexplained falls presenting to an emergency department. Insertion of an ILR (Reveal, Medtronic, Minnesota, USA) was used to detect arrhythmia. The primary outcome was detection of cardiac arrhythmia associated with a fall or syncope. The secondary outcome was detection of cardiac arrhythmia independent of falls or syncope, and falls or syncope without associated arrhythmia.

Results Seventy patients, mean age 70 years (5185 years) received an ILR. In $70 \%$ of patients cardiac arrhythmias were detected at a mean time of 47.3 days (SD 48.25). In $20 \%$, falls were attributable to a modifiable cardiac arrhythmia; 10 (14\%) received a cardiac pacemaker, 4 (6\%) had treatment for supraventricular tachycardia. Patients who had a cardiac arrhythmia detected were more likely to experience a further fall.

Conclusions 14 (20\%) patients demonstrated an arrhythmia which was attributable as the cause of their fall. Patients who have cardiac arrhythmia are significantly more likely to experience future falls. Further research is important to investigate if early detection of arrhythmogenic causes of falls using the ILR prevents future falls in older patients.

\section{INTRODUCTION}

Falls are the most common cause of injury and associated morbidity and mortality in older people. ${ }^{1}$ Due to accelerated growth of the global ageing demographic, the number of fall-related presentations to the emergency department (ED) has increased by $>50 \%$ in the last two decades. ${ }^{2}$ Falls are the single most common reason for older patients to attend the ED, accounting for one-third of all adult attendances. ${ }^{1}{ }^{3}$ Falls in older adults are more likely to be associated with significant injury, including hip fractures, and more likely to lead to hospital admission. ${ }^{4}$ Direct and indirect costs of falls are $>£ 2$ billion per year in the UK and US\$30 billion in the USA per year. ${ }^{15}$ Up to one-fifth of older fallers have no obvious cause for their fall event and are classified as 'unexplained' or 'non- accidental' falls. ${ }^{6}$ In the emergency setting the injury sustained following a fall is very often the primary focus of medical attention and important risk modification opportunities may be overlooked.

With advancing age cardiovascular morbidity plays an important role in the aetiology of syncope and unexplained falls. There is strong evidence of an overlap between syncope and unexplained falls in older people. Syncope is defined as transient loss of consciousness (T-LOC) due to transient global cerebral hypoperfusion characterised by rapid onset, short duration and spontaneous complete recovery. ${ }^{7}$ Up to $40 \%$ of older fallers have amnesia for loss of consciousness, and $60 \%$ of community dwelling older people have no witness to a fall event. ${ }^{8} 9$ Thus 'syncope' is likely to present as a fall in the absence of a witness account, coupled with amnesia for loss of consciousness. These patients are also more likely to sustain a serious injury. ${ }^{10}$

Falls have been associated with orthostatic hypotension $(\mathrm{OH})$, vasovagal syncope (VVS), carotid sinus syndrome (CSS) and cardiac arrhythmias, but attributing a single cause for syncope and unexplained falls is challenging and diagnostic clarity can be elusive. ${ }^{11}$ Recently, implantable loop recorders (ILRs) have greatly contributed to the diagnosis of arrhythmia as a cause of syncope. ${ }^{12}$ The most recent European Society of Cardiology (ESC) syncope guidelines recommend that ILR monitoring should be considered in patients with recurrent, unexplained syncope or falls after conventional work-up, ${ }^{13}$ although the evidence for the use of ILR monitoring in patients with unexplained falls is lacking.

We hypothesise that cardiac arrhythmias are an underdiagnosed cause of unexplained falls. The objective of this study is to determine the diagnostic utility of cardiac loop recorders in detection of arrhythmogenic causes for unexplained falls in older patients.

\section{METHODS}

A single centre, prospective, observational cohort study of recurrent fallers (defined as two or more falls in the previous year) was undertaken. All patients $>50$ years who presented to the ED as a result of an unexplained fall were screened during a 6-month period. A 'fall' was defined as an event which resulted in a person coming to rest inadvertently on the ground or floor or other lower level; ${ }^{14}$ an 'unexplained fall', as an event for which a cause was not apparent, either intrinsic (eg, stroke, myocardial infarction, gastrointestinal haemorrhage or 
other medical diagnosis) or extrinsic (eg, trip over carpet). ${ }^{15}$ All patients provided written informed consent and institutional ethics committee approval was obtained (reference number 2012/12/18).

Between the hours of 09:00 and 17:00, a research doctor and nurse screened all patients presenting to ED. ED records of patients presenting outside these hours were screened daily and eligible patients were contacted by telephone and invited to participate in the study. Patients with a life expectancy of $<12$ months, cognitive impairment (defined as a mini-mental state examination (MMSE) <24), ${ }^{16}$ a pacemaker in situ or prior diagnosis of a syncope syndrome were excluded. Patients with no access to a telephone landline were also excluded.

A comprehensive geriatric assessment was performed on all patients detailing falls history, fear of falling using the Falls Efficacy Scale (maximum score 0/100), ${ }^{17}$ medications history (classified according to the British National Formulary), polypharmacy (defined as five or more drugs), timed up and go test (TUG) (abnormal score $13.5 \mathrm{~s}){ }^{18}$ balance and mobility assessment (performance oriented mobility assessment (POMA)) (maximum score 56), ${ }^{19}$ mental health scores (Centre for Epidemiologic Studies Depression Scale) (maximum score 60) ${ }^{20}$ and medical history (self-report, doctor diagnosed). Where risk factors for falls were detected, falls prevention interventions were delivered in keeping with current guidelines prior to insertion of ILR. ${ }^{21}$

Cardiovascular assessment was undertaken in line with ESC guidelines, ${ }^{7}$ and included a 12-lead ECG, phasic blood pressure (BP) and heart rate (HR) measurement during orthostatic change from supine (10 min resting period) to upright over a 3 min period (active stand) with ECG and phasic BP recordings (BeatScope Finometer data). Carotid sinus massage (supine left and right and upright position at $70^{\circ}$ ), and head-up tilt-table test were performed when indicated. ${ }^{22}$ Patients with $\mathrm{OH}$, vasodepressor carotid sinus hypersensitivity and suspected VVS had appropriate interventions. Patients diagnosed with cardioinhibitory carotid sinus syndrome (CICSS), sinus bradycardia, first, second or third degree atrioventricular block (AVB), supraventricular tachycardia (SVT) were withdrawn from the study and appropriate treatment instituted (figure 1).
Figure 1 Flow diagram for patient screening. ILR, implantable loop recorder; SVT, supraventricular tachycardia.

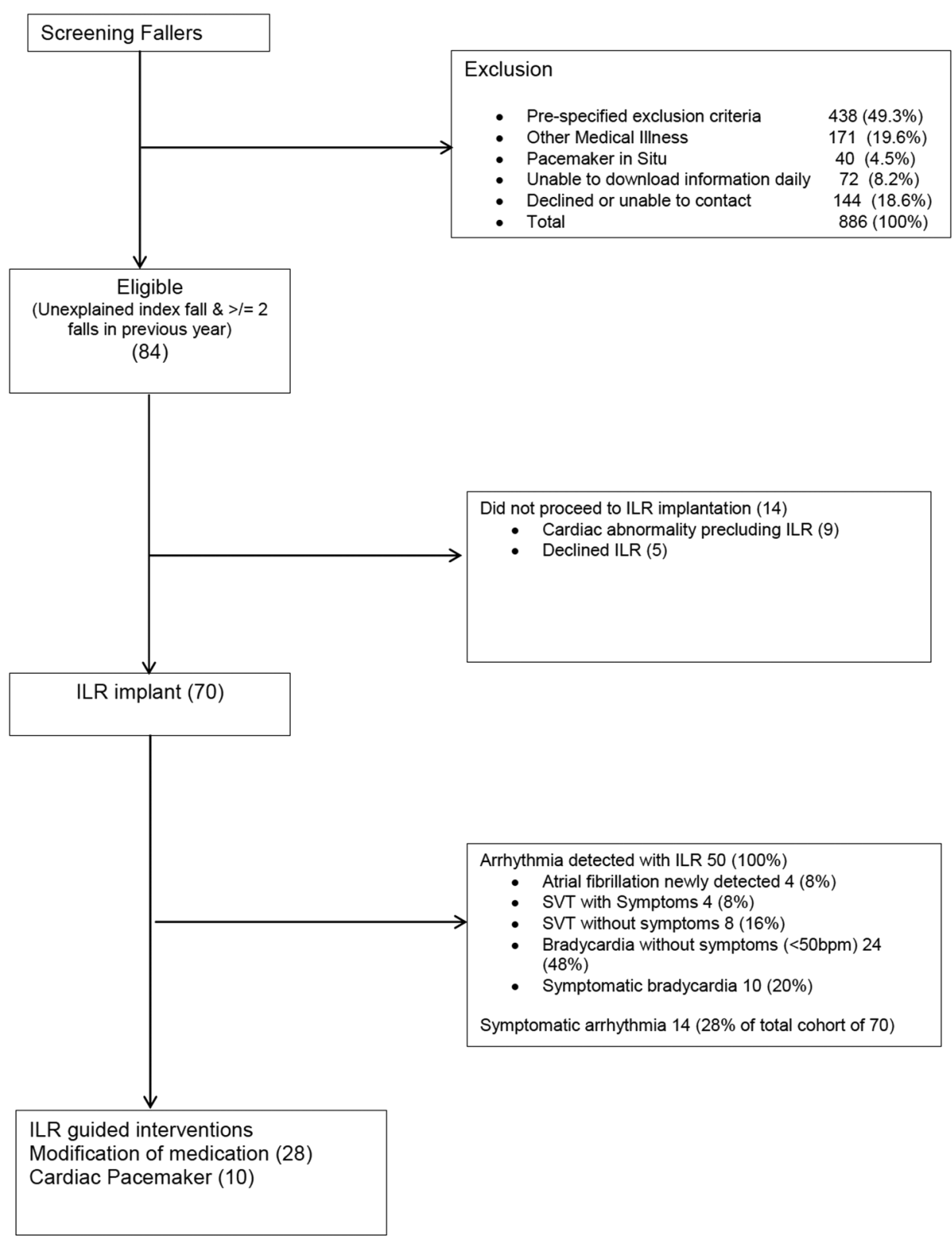




\section{Implantable loop recorder}

Remaining consented patients underwent ILR (Reveal, Medtronic, Minnesota, USA) implantation (figure 1). The ILR device was implanted in the left parasternal region, under aseptic conditions. It is capable of storing ECG data automatically in response to a significant bradycardia or tachyarrhythmia and in response to patient activation. The ILR DX model was used in the initial stage of the study and the ILR XT model in the latter stage when it became available, as it is a superior model for detection of atrial fibrillation. ECG data were downloaded and interpreted remotely on a daily basis using the CARELINK system (Medtronic) with all patient activations as well as preprogrammed alerts reviewed. Patients were instructed to activate the device after syncope, presyncope or fall. Any cardiac arrhythmias detected during the study were treated as appropriate (figure 1).

Participants returned weekly symptom diaries, with regular telephone prompting (biweekly) to optimise compliance. Details on the circumstance of each fall or syncopal event together with prodromal symptoms, consequences (ie, fracture, head injury) and hospital and ED attendances were recorded. Patients who had a subsequent fall during the trial were reviewed by a member of the investigating team and modifiable risk factors were treated at that time. Patients also attended for scheduled clinical review at six monthly intervals. The minimum follow-up was 6 months.

The primary outcome measure was detection of cardiac arrhythmia associated with a fall or syncope after implantation of the ILR. Cardiac arrhythmias detected by the ILR were defined according to ISSUE classification. ${ }^{23}$ The secondary outcome measure was (a) detection of cardiac arrhythmia independent of falls or syncope after implantation of the ILR and (b) a subsequent fall or syncope episode without associated arrhythmia after initial clinical assessment and implantation of ILR.

\section{Statistical analysis}

SAS software (V.9.3) was used for the calculation of sample size requirements with the Clopper-Pearson exact binomial method using a two-sided 95\% CI. The expected proportion of patients, who have an arrhythmia within 1 year, was 0.33 . This estimate was based on previous studies that used a sample size of 200 to detect an $18 \%$ improvement in ECG diagnosis. ${ }^{24}$ Under these assumptions, a sample size of 45 subjects with 1-year follow-up was required for the evaluation of this objective. Assuming an attrition rate of $10 \%$, a minimum sample size of 50 subjects was required.

Means and SDs or number and percentages were calculated for patients' baseline characteristics. Patient characteristics including the number and percentage use of medicines, and polypharmacy between the arrhythmia and non-arrhythmia group were compared using Fisher's exact test, with significance at $\mathrm{p}<0.05$ assumed. Student's $\mathrm{t}$ test was used for continuous variables.

\section{Screening}

A total of 970 ED fallers were screened over the study period (figure 1). In that 84 patients were eligible for study inclusion as they had presented to ED because of an unexplained fall, had at least two falls in the previous year and an MMSE > 24 (figure 1). Of these a cardiac arrhythmia or conduction disorder was diagnosed at initial cardiovascular assessment in nine patients, and therefore they did not proceed to ILR implantation. Arrhythmias detected at this assessment included five CICSS, one VVS, two trifascicular block, one second degree AVB.

\section{RESULTS}

\section{Baseline characteristics}

Seventy patients underwent ILR implantation $183 \%$ of original screening cohort), mean age 70 years (SD \pm 10.02 , range 50 82 years), 45 females (63\%), median MMSE of 28 (range 2430 ). The mean number of falls in the last year was 4.17 (range 2-12) per patient. The mean follow-up period was 9 months (range 6-12 months) (table 1).

\section{Arrhythmia}

Fifty patients (71.4\%) had a cardiac arrhythmia detected by ILR at a mean of 47.3 days (SD 48.25, range 1-190 days) postimplantation (table 2). Fourteen (28\%) met the primary end point of simultaneous fall or syncope together with a novel cardiac arrhythmia. The mean time to event in these patients was 43 days (SD 36.28). Cardiac pacing for bradycardia or asystole was required in $10(20 \%)$ and treatment of SVT in $4(8 \%)$ in order to prevent further falls. A further 24 patients had medication modification as a result of arrhythmias detected by the ILR. Patients who had an arrhythmia detected were more likely to have a history of comorbid diagnoses including cardiovascular disease, hypertension, depression, arthritis and hypercholesterolaemia. They were also more likely to be on five or more medications and to have suffered injurious events in their index fall (prior to enrolment into the study) (table 1).

\section{Secondary end points}

Cardiac arrhythmia independent of falls or syncope was detected in 36 (51\%) patients. These included atrial fibrillation, SVT and sinus bradycardia of $<50 \mathrm{bpm}$ which were detected in $4(8 \%), 8(16 \%)$ and $24(48 \%)$ patients, respectively, independent of falls or syncope. Thirty-six $(51 \%)$ patients had a fall or T-LOC during follow-up which was not associated with an arrhythmia; despite application of guideline-based treatment for prevention of falls. Mean time to first falls or T-LOC was 93.33 days (SD 72.15) days after implant. Patients who had a cardiac arrhythmia detected by the ILR 30 (83\%) were more likely to fall during follow-up $(p=0.0012)$ than those who did not (table 3 ). One patient had a witnessed seizure and was subsequently diagnosed with epilepsy. One patient had a fall that resulted in a hip fracture.

\section{DISCUSSION}

A major finding in this study is that 50 (71.4\%) of older patients with unexplained falls who attend the ED have a cardiac arrhythmia that is not apparent at the time of presentation, but detected within 9 months of continuous monitoring using ILR. In $14(20 \%)$ of these patients, events were directly attributable to a modifiable cardiac arrhythmia. A further $9(11 \%)$ have an arrhythmia detected during the initial detailed cardiovascular assessment. Furthermore, falls were more likely to recur in patients who had cardiac arrhythmias.

We have recently demonstrated in a large population study, the Irish longitudinal study of ageing (TILDA), that cardiovascular diseases, including cardiac arrhythmias such as atrial fibrillation, are retrospectively and prospectively associated with falls risk. ${ }^{25}$ Additionally, previous studies of ED cohorts support an association between falls and cardiovascular disorders such as $\mathrm{OH}, \mathrm{VVS}$ and CSS and to a lesser extent cardiac arrhythmias. ${ }^{6}$ This study has demonstrated that cardiac arrhythmias occurred with a large frequency in this cohort; with 50 (71.4\%) of patients having an arrhythmia detected. There were $14(28 \%)$ major arrhythmias detected at the time of a subsequent fall which resulted in prompt, ILR guided intervention. Previous 
Table 1 Baseline characteristics of patients with ILR inserted

\begin{tabular}{|c|c|c|c|c|}
\hline Variable & $\begin{array}{l}\text { Patients with } \\
\text { ILR inserted } \\
\mathrm{n}=70\end{array}$ & $\begin{array}{l}\text { Patients with } \\
\text { arrhythmia detected } \\
n=50\end{array}$ & $\begin{array}{l}\text { Patients with } \\
\text { no arrhythmia detected } \\
n=20\end{array}$ & p Value \\
\hline Sex (female) & $45(63 \%)$ & $29(58 \%)$ & $16(80 \%)$ & 0.10 \\
\hline Mean age \pm SD (years) & $69.4 \pm 10.0$ & $67.9 \pm 10.24$ & $72.4 \pm 8.38$ & 0.07 \\
\hline MMSE score* $†($ mean $\pm S D)$ & $28.18 \pm 1.746$ & $28.28 \pm 1.71$ & $27.94 \pm 1.82$ & 0.49 \\
\hline TUG score $\ddagger($ mean \pm SD) & $14.1 \pm 4.47$ & $13.3 \pm 3.92$ & $15.13 \pm 4.935$ & 0.39 \\
\hline CES-D score§ (mean \pm SD) & $9 \pm 8.001$ & $11.08 \pm 8.02$ & $8.167 \pm 7.53$ & 0.23 \\
\hline POMAף (mean \pm SD) & $24.76 \pm 4.02$ & $25.10 \pm 3.908$ & $26 \pm 4.11$ & 0.455 \\
\hline $\mathrm{FES}^{* *}($ mean $\pm \mathrm{SD})$ & $22.39 \pm 22.65$ & $22 \pm 21.85$ & $25.71 \pm 24.5$ & 1.00 \\
\hline Warning symptoms prior to index fall $\dagger$ & $21(30 \%)$ & $16(32 \%)$ & $5(25 \%)$ & 0.77 \\
\hline Head injury during index fall & $18(26 \%)$ & $10(14 \%)$ & $8(40 \%)$ & 0.801 \\
\hline Fracture during index fall $\ddagger \ddagger$ & $24(34 \%)$ & $16(23 \%)$ & $8(40 \%)$ & 0.115 \\
\hline History of cardiovascular disease $\S$ & $31(44 \%)$ & $27(39 \%)$ & $2(10 \%)$ & $0.001^{*}$ \\
\hline Hypertension & $41(59 \%)$ & $32(46 \%)$ & $9(45 \%)$ & $<0.001^{*}$ \\
\hline Diabetes & $8(11 \%)$ & $7(10 \%)$ & $1(5 \%)$ & 0.062 \\
\hline Hypercholesterolaemia & $32(46 \%)$ & $27(39 \%)$ & $5(25 \%)$ & $<0.001$ * \\
\hline COPD & $11(16 \%)$ & $9(13 \%)$ & $2(10 \%)$ & 0.055 \\
\hline Depression & $20(29 \%)$ & $18(36 \%)$ & $2(10 \%)$ & $<0.001$ * \\
\hline Osteoporosis & $15(21 \%)$ & $9(18 \%)$ & $6(30 \%)$ & 0.586 \\
\hline Polypharmacy ( $>5$ medications) & $41(59 \%)$ & $28(56 \%)$ & $13(65 \%)$ & 0.06 \\
\hline Antihypertensive & $44(63 \%)$ & $32(64 \%)$ & $12(60 \%)$ & $<0.001^{*}$ \\
\hline Antiarrhythmic & $7(10 \%)$ & $7(14 \%)$ & $0(0 \%)$ & 0.013 \\
\hline$\beta$-blockers & $15(21 \%)$ & $12(24 \%)$ & $3(15 \%)$ & 0.026 \\
\hline Hypnotic/anxiolytic & $16(23 \%)$ & $12(24 \%)$ & $4(20 \%)$ & 0.064 \\
\hline Antidepressant & $22(31 \%)$ & $16(32 \%)$ & $6(30 \%)$ & $0.001^{*}$ \\
\hline Non-opioid analgesia & $15(21 \%)$ & $11(22 \%)$ & $4(20 \%)$ & 0.098 \\
\hline Diuretic & $12(17 \%)$ & $10(20 \%)$ & $2(10 \%)$ & 0.030 \\
\hline Antiplatelet & $25(36 \%)$ & $18(36 \%)$ & $7(35 \%)$ & 0.025 \\
\hline Bisphosphonate & $10(14 \%)$ & $5(10 \%)$ & $5(25 \%)$ & 1.00 \\
\hline \multicolumn{5}{|c|}{ 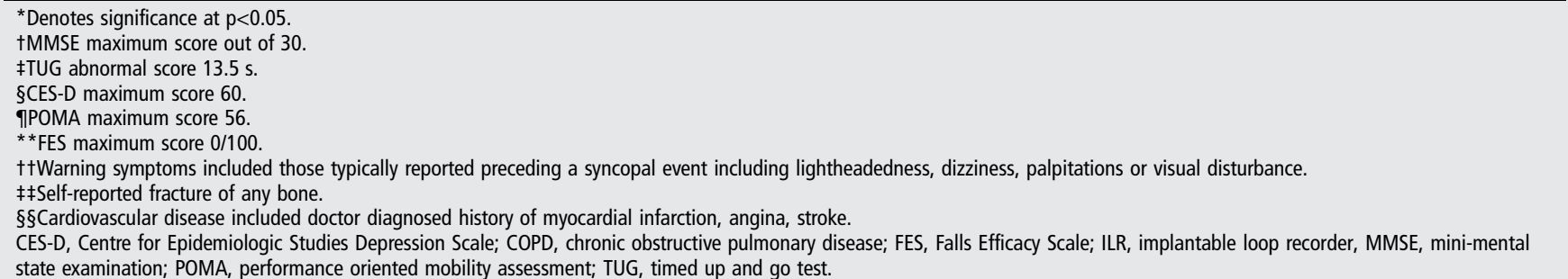 } \\
\hline
\end{tabular}

observational studies have shown a prevalence between $1 \%$ and $25 \%$ for detection of cardiac arrhythmia in older fallers. ${ }^{26}$ They differed significantly in their methods, definitions of cardiac arrhythmia and the ways in which they had captured

Table 2 Description and classification of arrhythmias detected in patients with ILR inserted at a mean of 9 months

\begin{tabular}{llll}
$\begin{array}{l}\text { Number of } \\
\text { patients } \\
50(100 \%)\end{array}$ & $\begin{array}{l}\text { Mean } \\
\text { age } \pm \text { SD }\end{array}$ & Description of arrhythmia & $\begin{array}{l}\text { ISSUE } \\
\text { classification }\end{array}$ \\
\hline $9(18 \%)$ & $73 \pm 9.04$ & $\begin{array}{l}\text { Asystole (RR interval pause } \geq 3 \mathrm{~s}) \\
\text { Bradycardia (HR }<40 \mathrm{bpm} \text { for } \\
>10 \mathrm{~s})\end{array}$ & 2 \\
$22(44 \%)$ & $67.45 \pm 10.23$ & $\begin{array}{l}\text { Bradycardia (HR }>40 \mathrm{bpm} \text { and } \\
<60 \text { bpm for }>10 \mathrm{~s})\end{array}$ & 3 \\
$15(30 \%)$ & $65.26 \pm 10.38$ & \begin{tabular}{l} 
SVT $(>140 \mathrm{bpm}$ for $>15 \mathrm{~s})$ \\
\hline
\end{tabular}
\end{tabular}

HR, heart rate; ILR, implantable loop recorder; SVT, supraventricular tachycardia. arrhythmia. This is the first study to look at prolonged cardiac monitoring in a large group with prospective falls diaries coupled with capture of abnormal cardiac rhythms. We have provided evidence which strengthens the association between cardiac arrhythmias and falls and demonstrated that cardiac arrhythmias are a casual, modifiable risk factor in falls prevention.

Previous studies using prolonged monitoring for detection of cardiac arrhythmias has focused on syncope as a primary outcome and has supported the use of ILR in older adults. Brignole et al previously compared the use of ILR in patients over the age of 65 to those under the age of 65 , referred for investigation of unexplained syncope. Syncope recurrence was 2.7 times higher and modifiable cardiac arrhythmias were 3.1 times more frequent in those $>65$ years. ${ }^{27}$ The diagnostic yield of ILR is higher in older patients; use of an ILR in older fallers achieved a diagnostic yield similar to that reported for syncope at $20 \%$. Furthermore, cardiac data were obtained as a result of ILR monitoring which resulted in detection of asymptomatic cardiac arrhythmias which did not require an invasive 
Table 3 Univariate analysis of patients with an ILR inserted who experienced further falls during a mean follow-up of 9 months

\begin{tabular}{llll}
\hline Fall risk factor at baseline & $\begin{array}{l}\text { Fall during } \\
\text { follow-up } \\
\mathrm{N}=36\end{array}$ & $\begin{array}{l}\text { No fall during } \\
\text { follow-up } \\
\mathrm{N}=34\end{array}$ & $\mathbf{p}$ Value \\
\hline Age & $68.2(10.80)$ & $70.2(8.86)$ & 0.40 \\
TUG (mean \pm SD) & $11.39 \pm 1.56$ & $10.60 \pm 1.24$ & 0.42 \\
MMSE (mean \pm SD) & $28.27 \pm 0.60$ & $28.08 \pm 0.60$ & 0.65 \\
CES-D (mean \pm SD) & $12.47 \pm 2.18$ & $8.79 \pm 2.58$ & 0.06 \\
POMA (mean \pm SD) & $24.41 \pm 1.604$ & $25.09 \pm 1.12$ & 0.49 \\
FES (mean $\pm S D)$ & $25.5 \pm 8.19$ & $19.35 \pm 6.85$ & 0.25 \\
Arrhythmia detected during & $30(83 \%)$ & $6(18 \%)$ & $0.001^{*}$ \\
follow-up & & & \\
$36(100 \%)$ & & & \\
\hline
\end{tabular}

*Denotes significance at $p<0.05$.

CES-D, Centre for Epidemiologic Studies Depression Scale; FES, Falls Efficacy Scale;

ILR, implantable loop recorder; MMSE, mini-mental state examination; POMA,

performance oriented mobility assessment; TUG, timed up and go test.

procedure, but resulted in guided treatment interventions. For example, four patients had new atrial fibrillation detected requiring the initiation of anticoagulation and a further 24 patients had medication dosages adjusted because of the detection of cardiac arrhythmia. Our data support an initial detailed cardiovascular assessment in patients with unexplained falls and also continuous prolonged cardiac monitoring using ILR to detect underlying cardiac arrhythmias.

This study has demonstrated that those patients with cardiovascular disease, hypertension and hypercholesterolaemia were most likely to have cardiac arrhythmia detected by ILR. In addition, those with higher depression scores as well as arthritis had a higher risk of detection of cardiac arrhythmia demonstrating the multifactorial nature of falls in older adults. Our results support the American Geriatric Society (AGS)/British Geriatric Society (BGS) and National Institute for Health and Care Excellence (NICE) guidelines, which recommend standardised cardiovascular investigations as well as a multifactorial assessment in all patients with recurrent falls in order to detect and adequately prevent future falls. ${ }^{21}{ }^{28}$ Overall $51 \%$ of the cohort had a subsequent fall during follow-up despite application of guideline-based falls assessment and intervention emphasising that these are high-risk patients for whom new interventions are needed. ${ }^{21}$ Randomised control trials, which include multifactorial intervention for traditional falls risk factors, coupled with targeted treatment of cardiovascular disorders show benefit for falls prevention in cognitively intact older patients. ${ }^{29}$ In one study, dual-chamber cardiac pacing reduced falls by $70 \%$ during a 12-month follow-up period in patients with unexplained falls and CSS. $^{29}$ Despite this and other evidence, cardiovascular assessments are not consistently performed in ED. ${ }^{30}$ Further randomised studies are needed to discern if targeted intervention of arrhythmia is of benefit for falls reduction in older adults.

One explanation for the overlap between syncope and falls is amnesia for loss of consciousness. ${ }^{8}$ If patients with cardiac arrhythmia have amnesia for T-LOC and if events are not witnessed, the patient will present with an unexplained fall rather than syncope. In this study, the detection of asymptomatic arrhythmia was a common finding with the majority of arrhythmias detected not occurring at the time of a fall or collapse episode. Furthermore, bradycardia detected $<50 \mathrm{bpm}$ but $>40 \mathrm{bpm}$ was the most common type of arrhythmia described.
Although these HRs are often considered a normal variant; patients with this arrhythmia were more likely to have suffered a subsequent fall than those who maintained a normal sinus rhythm. In addition, commonly measured variables predictive of gait imbalance and falls risk such as TUG and POMA scores were not significantly higher in the falls group. In the absence of concomitant BP measurement, it is difficult to ascertain the exact clinical consequences of intermittent arrhythmia. One explanation is that modest reductions in cerebral perfusion are caused by hypotension secondary to arrhythmia, resulting in balance instability and consequent falls without necessarily causing loss of consciousness. ${ }^{7}$ Another plausible explanation is that repeated episodes of arrhythmia are sufficient to result in cerebral hypoperfusion and vascular damage to neural pathways which govern balance. Further research looking at the effect of intermittent arrhythmia on BP and cerebral perfusion may provide insight into optimal HR management in older patients with falls.

\section{LIMITATIONS}

This is a single site prospective observational cohort study. In this series, $10 \%$ of patients $>50$ years who attended ED because of a fall were classified as unexplained. This is likely to be an underestimation of the true prevalence of this condition. We excluded patients who were cognitively impaired or who were in an institution and did not have access to a landline. The prevalence of cardiac arrhythmia in these cohorts has not been studied. The implication of our findings in other settings, that is, community falls without injury, requires separate study. It may be that injurious falls are more likely to be associated with arrhythmias. A multicentre trial to determine whether the findings can be generalised and whether ILR guided intervention prevents falls is now warranted.

\section{CONCLUSION}

A better understanding of causal factors for unexplained falls is critical in order to develop more effective prevention strategies and improve successful ageing in our changing population demographic. Further studies are now required to determine whether ILR guided intervention coupled with traditional risk factor modification will prevent falls in older patients.

\section{Key messages}

What is already known on this subject?

Unexplained falls account for $20 \%$ of falls in older cohorts. The role of the implantable loop recorder in the detection of arrhythmias in patients with unexplained falls is unknown.

\section{What might this study add?}

Twenty per cent of unexplained fallers demonstrate an arrhythmia which is attributable as the cause of their fall. Patients who have cardiac arrhythmia are significantly more likely to experience future falls.

\section{How might this impact on clinical practice?}

In this paper, we describe a novel approach to assessment of falls risk using an implantable loop recorder and show that cardiac arrhythmias have a high prevalence in older unexplained fallers. 
Contributors All authors contributed to the production of this manuscript and the conduct of this trial.

Competing interests This trial was partly funded by Medtronic which provided the devices used for the trial.

Ethics approval St James's Hospital.

Provenance and peer review Not commissioned; externally peer reviewed.

Data sharing statement All additional unpublished information is kept on a secure server in the institution and only available to the first and senior author of the manuscript.

\section{REFERENCES}

1 CDC-Older Adult Falls_-Falls Among Older Adults: An Overview-Home and Recreational Safety-Injury Center. 2014. http://www.cdc.gov/ homeandrecreationalsafety/falls/adultfalls.html

2 Hartholt KA, van der Velde N, Looman CW, et al. Trends in fall-related hospital admissions in older persons in the Netherlands. Arch Intern Med 2010;170:905-11.

3 Albert M, McCaig LF, Ashman JJ. Emergency department visits by persons aged 65 and over: United States, 2009-2010. NCHS Data Brief 2013;130:1-8.

4 Close JCT, Lord SR, Antonova EJ, et al. Older people presenting to the emergency department after a fall: a population with substantial recurrent healthcare use. Emerg Med J. 2012;29:742-7.

5 Tian Y, Thompson J, Buck D. et al. Exploring the system-wide costs of falls in older people in Torbay. London: The King's Fund. 2013.

6 Davies AJ, Kenny RA. Falls presenting to the accident and emergency department: types of presentation and risk factor profile. Age Ageing 1996;25:362-6.

7 Moya A, Sutton R, Ammirati F, et al. Guidelines for the diagnosis and management of syncope (version 2009). Eur Heart J 2009;30:2631-71.

8 Parry SW, Steen IN, Baptist M, et al. Amnesia for loss of consciousness in carotid sinus syndrome: implications for presentation with falls. J Am Coll Cardiol 2005;45:1840-3.

9 Cummings SR, Nevitt MC, Kidd S. Forgetting falls. The limited accuracy of recall of falls in the elderly. J Am Geriatr Soc 1988;36:613-6.

10 Nevitt MC, Cummings SR, Hudes ES. Risk factors for injurious falls: a prospective study. J Gerontol 1991;46:M164-70.

11 Sutton R, Brignole M, Benditt DG. Key challenges in the current management of syncope. Nat Rev Cardiol 2012;9:590-8.

12 Brignole $M$, Vardas $P$, Hoffman $E$, et al. Indications for the use of diagnostic implantable and external ECG loop recorders. Europace 2009;11:671-87.

13 Brignole M, Auricchio A, Baron-Esquivias G, et al. 2013 ESC guidelines on cardiac pacing and cardiac resynchronization therapy: the task force on cardiac pacing and resynchronization therapy of the European Society of Cardiology (ESC). Developed in collaboration with the European Heart Rhythm Association (EHRA). Europace 2013;15:1070-118.
14 WHO|Falls. WHO, 2014.

15 Parry SW, Steen N, Bexton RS, et al. Pacing in elderly recurrent fallers with carotid sinus hypersensitivity: a randomised, double-blind, placebo controlled crossover trial. Heart 2009;95:405-9.

16 Folstein MF, Robins LN, Helzer JE. The mini-mental state examination. Arch Gen Psychiatry 1983:40:812.

17 Tinetti ME, Richman D, Powell L. Falls efficacy as a measure of fear of falling. J Gerontol. 1990;45:P239-43.

18 Podsiadlo D, Richardson S. The timed "Up \& Go": a test of basic functional mobility for frail elderly persons. J Am Geriatr Soc 1991;39:142-8.

19 Tinetti ME. Performance-oriented assessment of mobility problems in elderly patients. J Am Geriatr Soc 1986;34:119-26.

20 Weissman MM, Sholomskas D, Pottenger M, et al. Assessing depressive symptoms in five psychiatric populations: a validation study. Am J Epidemiol 1977; 106:203-14.

21 Panel on Prevention of Falls in Older Persons, American Geriatrics Society and British Geriatrics Society. Summary of the Updated American Geriatrics Society/ British Geriatrics Society clinical practice guideline for prevention of falls in older persons. J Am Geriatr Soc 2011;59:148-57.

22 Bartoletti A, Alboni P, Ammirati F, et al. 'The Italian Protocol': a simplified head-up tilt testing potentiated with oral nitroglycerin to assess patients with unexplained syncope. Europace 2000;2:339-42.

23 Brignole M, Moya A, Menozzi C, et al. Proposed electrocardiographic classification of spontaneous syncope documented by an implantable loop recorder. Europace 2005;7:14-8.

24 Farwell DJ, Freemantle N, Sulke N. The clinical impact of implantable loop recorders in patients with syncope. Eur Heart J 2006;27:351-6.

25 Jansen S, Kenny RA, de Rooij SE, et al. Self-reported cardiovascular conditions are associated with falls and syncope in community-dwelling older adults. Age Ageing 2015;44:525-9.

26 Jansen S, Bhangu J, de Rooij S, et al. The association of cardiovascular disorders and falls: a systematic review. J Am Med Dir Assoc 2015.

27 Brignole M, Menozzi C, Maggi R, et al. The usage and diagnostic yield of the implantable loop-recorder in detection of the mechanism of syncope and in guiding effective antiarrhythmic therapy in older people. Europace. 2005;7:273-9.

28 Centre for Clinical Practice at N. National Institute for Health and Clinical Excellence: Guidance. Falls: Assessment and Prevention of Falls in Older People. London: National Institute for Health and Care Excellence (UK)Copyright (c) National Institute for Health and Care Excellence. 2013.

29 Kenny RA, Richardson DA, Steen N, et al. Carotid sinus syndrome: a modifiable risk factor for nonaccidental falls in older adults (SAFE PACE). J Am Coll Cardiol 2001;38:1491-6.

30 Daccarett M, Brignole M, Malasana GR, et al. Underevaluation of cardiovascular risk factors in patients with nonaccidental falls. J Prim Care Community Health 2011;2:173-80. 